\title{
Berichtigung.
}

In seiner in diesem Archiv (Bd. 67. No. 2) erschienenen Arbeit "Ueber Myomotomie mit retroperitonealer Stielversorgung" will Heinricius feststellen, dass ich in dem Aufsatz: Myomoperationen, Statistik der Gynäkologie von Sänger und von Herff 1900 irrige Angaben bezüglich der von ihm ausgeführten Operationen gemacht habe und will diese als einen Beweis dafür ansehen, wie unzuvorlässig solche auf Grund von Zusammenstellungen von verschiedenen Seiten aufgestellten Gross-Statistiken sind. Ich bemerke hierzu Folgendes:

1. Herr Prof. Heinricius citirt meine Angaben selbst falsch, was man nicht erwarten sollte, da derselbe gegen mich den Vorwurf falschen Citates erhebt: unter der Rubrik III, 2 anf Seite 90 der Encyklopädie: Supravaginale Hysterectomie mit extraperitonealer Stielversorgung steht Heinricius mit 5 Fällen mit $1 \div$ verzeichnet und nicht mit 24 Fällen mit $2 \dagger$; wie er angiebt.

2. Wie Heinricius richtig annimmt, entstammen meine Angaben der Arbeit seines Schülers Granfelt in Bd. 33 der Finska Läkaresällskapets Handlingar. Da mir das Original nicht zur Verfügung stand, habe ich die Referate im Jahresbericht über Geburtsbülfe und Gynäkologie 1896 und im Centralblatt für Gynäkologie 1897 benutzt, welche beide ron Leopold Meyer verfasst sind und zwar in folgender Weise: Die 3 abdominalen Totalexstirpationen habe ich nicht für die Statistik verwerthet, weil jch durchweg nur Reiben von mindestens 5 Operationen nach einer Methode eingesetzt habe, da m. E. einzelne Fälle für eine grössere Statistik ohne Werth sind. Eine Ausnahme ist hierin nur bei der „Unterbindung der Blut. zuführenden Gefässe" gemacht, weil diese Methode ausser von den Autoren, die sie angegeben haben, nur von einigen wenigen andern Operateuren ausgefübrt ist. Die 15 abdominalen Enucleationen mit $2 \uparrow$ and 18 Abtragungen subseröser Geschwülste mit $3 \leftarrow$ sind unter der Rubrik: Abdominale Enueleation zusammengefasst und zwar aus dem dort erwähnten Grunde, 
weil manche Operateure auch die Entfernung gestielter Myome den Enucleationen beizählen und deswegen eine schroffe Trennung beider Operationsarten für die Statistik kaum möglich ist. Die 5 supravaginalen Hysterectomien nach Péan mit $1 \dagger$ sind unter die supravaginalen Hysterectomien mit extraperitonealer Stielversorgung eingereiht. In der Abtheilung: Supravaginale Hysterectomie mit intraperitonealer Stielversorgung sind zunächst die 18 Operationen nach Schröder mit $5 \dagger$, der eine Fall von Versenkung des Stumpfes mit elastischer Schlinge mit $1 \dagger$ und die 24 Operationen nach Chrobak mit $2 \div$ zusammengefasst (43 mit $8 \uparrow$ ); letztere sind dann nochmals gesondert aufgeführt unter denjenigen Hysterectomien mit intraperitonealer Stielversorgung, die nach retroperitonealer Methode ausgeführt wurde. Die 20 Castrationen mit $1 \dot{\dagger}$, die 10 vaginalen Enucleationen obne $\uparrow$ sind genau wie im Referat angegeben, aufgeführt. Da im Referate L. Meyer's bemerkt ist, dass die meisten Operationen von Heinricius ausgeführt worden seien, nahm ich keinen Anstand, sie unter seinem Namen aufzuführen, ebenso wie das bei anderen Klinikvorständen geschieht, obwohl eine Reihe der ihnen zugerechneten Operationen von andern Aerzten (Assistenten u. s. w.) vollzogen wurden."

Ich glaube bewiesen zu haben, dass Heinricius' Behauptung, dass meine Angaben irrig seien, vollständig unberechtigt ist, und dass daraus keinerlei Schlüsse auf Unzuverlässigkeit meiner Statistik gezogen werden können. Ich bemerke, dass ich, soweit es möglich war, die Originalarbeiten durchgesehen habe und für die übrigen Fälle die Referate in den verschiedenen Fachzeitschriften mit einander verglichen habe, um zu möglichst zuverlässigen Zahlen zu kommen. Dass das Referat L. Meyer's correct ist, hat Heinricius selbst anerkannt. Was ich vom Werth einer solchen grossen Statistik halte, habe ich in der Einleitung des betreffenden Artikels in der Encyklopädie auseinandergesetzt. Die dort niedergelegte Ansicht wird wohl von der Fachpresse allgemein getheilt werden. Chemnitz, den 8. Oktober 1902.

\section{Dr. Praeger.}

Durch das Entgegenkommen der Redaction bin ich in der Lage gewesen, die Widerlegung meines Collegen Praeger über die von mir gemachte Anmerkung seiner Statistik der Myomoperationen durchzulesen, so weit dieselbe die auf der gynäkologischen Universitätsklinik in Helsingfors ansgeführten Operationen betrifft. 
So wie College Praoger die nach verschiedenen Hothoden ausgeführon Operationen zusammengefasst hat, kommt man wirklich zu der Zahl, die er angegeben. In dieser Beziehung sind die Angaben von $P$. nicht irrig:

Praeger hat die Fälle von Abtragung gestielter Myome mit den Fällen von Enucleation der Myome per laparatomiam zusammengestellt und auf diese Weise hat er 33 abdominale Enucleationen bekommen, "weil eine scharfe Trennung beider Operationsarten für die Statistik kaum möglich ist". Meiner Ansicht nach sind doch die Enucleation der gewöhnlich interstitiellen Vyome und die Entfernung gestielter Myome verschiedene Operationen.

College Praeger hat weiter als supravaginale Hysterectomien mit intraperitonealer Stielversorgung die 18 Operationen nach Schroeder, 1 Fall von Versenkung des Stumpfes mit elastischer Schlinge und 24 Operationen mit retroperitonealer Bebandlung des Stumpfes nach Chrobak zusammengestellt und auf diese Weise die Zahl 43 bekommen. Ich halte die Myomotomie mit intra- und extraperitonealer Stielversorgung als verschiedene Operationsmethoden.

Die Zusammenstellung Praeger's der in meiner Klinik ausgeführten Myomotomien stimmt nicht mit den Referaten im Centralblatt für Gynäkologie und in Frommel's Jahresbericht überein. Es kann wohl Niemanden verwundern, dass ich vor der Aufklärung Praeger's seine Zahl nicht äbereinstimmend mit der Angabe in obenerwähntem Referat des Originalaufsatzes Granfelt's fand.

Betreffs College Práeger's Behauptung, dass ich seine Angaben falsch citjrt bätte, da ich 24 Fälle von extraperitonealer Stielversorgung aufnahm, beruht der Fehler nur auf einer Nachlässigkeit beim Durchlesen (von mir) der Correctur. Im Manuscript steht 24 Fälle von retroperitonealer Stielversorgung; im Text ist retro verkehrt mit extra wiedergegeben. In einigen Zeilen vorher sind ja die 5 supravaginalen. Hysterectomien mit extraperitonealer Stielversorgung aufgenommen. Ein aufmerksamer Leser dürfte leicht finden, dass das "falsche" Citat auf einem Fehler in der Durchlesung der Correctur beruht.

Helsingfors, den 22. Nov. 1902.

Prof. G. Heinricius. 